\title{
Photoprotective effect of arctiin against ultraviolet B-induced damage in HaCaT keratinocytes is mediated by microRNA expression changes
}

\author{
HWA JUN CHA ${ }^{1}$, GHANG TAI LEE ${ }^{2,3}$, KWANG SIK LEE ${ }^{2,3}$, KUN KOOK LEE ${ }^{2}$, \\ JIN TAE HONG ${ }^{3}$, NA KYEONG LEE ${ }^{1}$, SOO-YEON KIM ${ }^{1}$, BO MI LEE ${ }^{1}$, IN-SOOK AN ${ }^{1}$, \\ HYUNG JIN HAHN ${ }^{4}$, KYU JOONG AHN ${ }^{4}$, SU-JAE LEE ${ }^{5}$, SUNGKWAN AN ${ }^{1}$ and SEUNGHEE BAE ${ }^{1}$ \\ ${ }^{1}$ Korea Institute for Skin and Clinical Sciences and Molecular-Targeted Drug Research Center, Konkuk University, \\ Seoul 143-701; ${ }^{2}$ Songpa R\&D Center, Coreana Cosmetics Co., Ltd., Cheonan, Chungcheongnam-do 330-833; \\ ${ }^{3}$ College of Pharmacy and Medical Research Center, Chungbuk National University, Cheongju, \\ Chungcheongbuk-do 361-763; ${ }^{4}$ Department of Dermatology, Konkuk University School of Medicine, Seoul 143-701; \\ ${ }^{5}$ Department of Chemistry, Hanyang University, Seoul 133-791, Republic of Korea
}

Received September 24, 2013; Accepted April 16, 2014

DOI: $10.3892 / \mathrm{mmr} .2014 .2326$

\begin{abstract}
Human keratinocytes are located in the outermost skin layer and thus particularly vulnerable to ultraviolet B (UVB) radiation exposure. Previous studies have focused on the cellular and molecular perspectives of UVB-induced keratinocyte damage. In the present study, it was demonstrated that pretreatment with the phytochemical arctiin, one of the lignin compounds, protects human $\mathrm{HaCaT}$ keratinocytes from UVB-mediated damage. Biochemical assays revealed that UVB-induced cytotoxicity and cell death were significantly reduced in arctiin-pretreated $\mathrm{HaCaT}$ cells. In addition, arctiin promoted the wound healing and DNA repair properties of keratinocytes. The photoprotective effects of arctiin were associated with changes in the expression levels of specific microRNAs (miRNAs) in HaCaT cells. A bioinformatics analysis demonstrated that the miRNAs were functionally involved in cancer, cell cycle, and Wnt and mitogen-activated protein kinase signaling pathways. In the present study, the results from the cellular and molecular assays demonstrated a novel role for arctiin in UVB protection in keratinocytes, which is mediated by miRNA responses and the suppression of UVB-induced cell death. Furthermore, arctiin is implicated as a potential chemopreventive agent through UVB protection of keratinocytes.
\end{abstract}

Correspondence to: Professor Seunghee Bae, Korea Institute for Skin and Clinical Sciences and Molecular-Targeted Drug Research Center, Konkuk University, 120 Neungdong-ro, Gwangjin-gu, Seoul 143-701, Republic of Korea

E-mail: sbae@konkuk.ac.kr

Key words: photoprotection, keratinocyte, ultraviolet B microRNA, arctiin

\section{Introduction}

Ultraviolet (UV) radiation induces several harmful effects, including DNA damage, reactive oxygen species (ROS) generation, cell cycle arrest, tumorigenesis, immunosuppression and apoptosis in skin cells (1). Therefore, photoprotection against UV-induced damage has an important role in maintaining skin health. UV radiation is classified as UVA, UVB and UVC. Although UVC is the most cytotoxic radiation, it is almost completely blocked by the ozone layer (1). UVA is a longer-wavelength radiation and is able to penetrate deep into skin (1). UVB is a shorter-wavelength radiation that scarcely penetrates into the dermis layer, but is more cytotoxic than UVA (1). UVB is considered to cause direct damage to DNA and indirect damage through the generation of ROS (1). Keratinocytes form the outermost tissue layer of the body and are therefore constantly exposed to UV radiation. Numerous studies have reported deleterious effects of UVB radiation on keratinocytes, including senescence, inflammation, cell death and epithelial malignancy (2). At the molecular level, UVB affects diverse signaling pathways in keratinocytes. Mitogen-activated protein kinases (MAPKs), including extracellular signal-regulated kinase (ERK), p38 MAPK, and c-Jun $\mathrm{N}$-terminal kinase (JNK), are among the major UVB response molecules in keratinocytes (2). UVB activates MAPKs to induce either apoptosis or inflammation (2). The nuclear factor kappa $\mathrm{B}(\mathrm{NF}-\kappa \mathrm{B})$ signaling pathway is also involved in UVB-mediated responses in keratinocytes (2). UVB-induced activation of NF- $\mathrm{BB}$ pathways induces the expression of several genes that regulate the cell cycle, apoptosis and inflammation (2).

Recently, microRNAs (miRNAs) have been revealed to have pivotal roles in differentiation, senescence, cell survival and apoptosis in keratinocytes. Rivetti et al (3) demonstrated that miRNA-p63 feedback is important for keratinocyte senescence. Hidebrand et al (4) analyzed miRNA expression during keratinocyte differentiation and identified a role of miRNAs 
in skin development, whereas Yang et al (5) demonstrated that miR-21 promotes keratinocyte migration and re-epithelialization during wound healing. Of note, it was recently reported that miRNAs are involved in UVB-mediated cellular and molecular responses in keratinocytes. Zhou et al (6) demonstrated that acute exposure of keratinocytes to UVB results in several specific patterns of miRNA response. Furthermore, Guo et al (7) revealed that protection of HaCaT keratinocytes against UVB radiation is mediated by miR-23a through the regulation of topoisomerase-1/caspase $7 /$ serine/threonine-protein kinase 4 (STK4). These results indicate that miRNAs are crucial regulators of keratinocyte development and UVB response.

Arctiin is a lignin compound that has been purified from several plants, including Arctium lappa and Forsythiae fructus. Previous studies have demonstrated that arctiin exerted a protective effect against lipopolysaccharide (LPS)-induced inflammation and has anti-proliferative and anti-microbial functions (8-11). However, to the best of our knowledge, the photoprotective effect of arctiin has not been studied. In the present study, it was demonstrated that treatment with a low dose of arctiin suppresses UVB-induced keratinocyte damage though specific changes in miRNA expression.

\section{Materials and methods}

Cell culture and chemicals. HaCaT keratinocytes were purchased from Cell Line Service (DKFZ, Eppelheim, Germany) and were maintained with Dulbecco's modified Eagle's medium (DMEM; Gibco, Life Technologies, Grand Island, NY, USA) supplemented with $10 \%$ fetal bovine serum (FBS; Gibco, Life Technologies) and antibiotics. Arctiin, a lignin isolated from Arctium lappa, was purchased from Sigma-Aldrich (St. Louis, MO, USA) and dissolved in dimethyl sulfoxide (DMSO; Sigma-Aldrich). Propidium iodide (PI), a fluorescent nuclear and chromosome counterstain, was purchased from BD Biosciences (San Jose, CA, USA).

Cytotoxicity assay. The cytotoxicity of arctiin on $\mathrm{HaCaT}$ cells was determined using a water-soluble tetrazolium (WST)-1 based cell viability assay (EZ-Cytox Cell Viability Assay kit; Itsbio, Seoul, Korea). HaCaT cells were seeded in a 96-well culture dish $\left(3 \times 10^{3}\right.$ cells/well) and grown for $24 \mathrm{~h}$ prior to the treatment with various doses of arctiin for a further $24 \mathrm{~h}$. At the end of the treatment, the cells were incubated with $10 \mu \mathrm{l}$ of the reagent from the WST-1 assay kit for $30 \mathrm{~min}$. Absorbance was recorded at $450 \mathrm{~nm}$ using a microplate spectrophotometer (iMark microplate reader; Bio-Rad, Hercules, CA, USA). The results are presented as the percentage of the control values.

$U V B$ protection assay. The UVB protective effect of arctiin in $\mathrm{HaCaT}$ keratinocytes was determined using a previously described method (12). Cells were pretreated with different concentrations of arctiin for various durations, washed with phosphate-buffered saline (PBS) and irradiated with UVB $\left(50 \mathrm{~mJ} / \mathrm{cm}^{2}\right)$ without lids on the plates. Following irradiation the cells were incubated for $24 \mathrm{~h}$ in growth media and cell viability was measured using an EZ-Cytox Cell Viability Assay kit (Itsbio). The results are presented as the percentage relative to the control (mean \pm standard deviation).
Cell cycle analysis using flow cytometry. Following exposure to UVB, the cells were washed twice with cold PBS and fixed by careful resuspension in cold $70 \%$ ethanol. Following fixation, the cells were washed with cold PBS and stained with PI solution $(0.05 \mathrm{mg} / \mathrm{ml}$ PI, $2 \mathrm{mg} / \mathrm{ml}$ RNase A, $0.1 \%$ Triton X-100 in PBS). Fluorescence intensity (FL-2H) was measured by flow cytometry (FACScalibur; BD Biosciences) with Cell Quest software (BD Biosciences).

In vitro scratch assay. Cell migration in vitro was measured using a scratch assay. Cells were seeded densely into $60 \mathrm{~mm}$ culture dishes. Confluent cells were treated with $\operatorname{arctiin}(5 \mu \mathrm{M})$ for $6 \mathrm{~h}$ and a standardized scratch was established in the monolayer using a $20 \mu \mathrm{l}$ loading tip. Following scratch formation the cells were irradiated with UVB $\left(50 \mathrm{~mJ} / \mathrm{cm}^{2}\right)$ and incubated in growth media. The extent of scratch closure was quantified by measuring the area of the scratch prior to $(0 \mathrm{~h})$ and $48 \mathrm{~h}$ following wounding, using a phase-contrast microscope (Olympus CKX41; Olympus, Tokyo, Japan).

DNA repair assay. The effect on arctiin on UV-damaged plasmid was determined using a luciferase system. The pGL3 luciferase vector (Promega Corporation, Madison, WI, USA) was irradiated with UVC at the dose rate of $200 \mathrm{~J} / \mathrm{m}^{2} / \mathrm{sec}$ for $10 \mathrm{sec}$ (total $2000 \mathrm{~J} / \mathrm{m}^{2}$ ). The undamaged control vector and UV-damaged pGL3 vector were co-transfected with pSV- $\beta$-galactosidase ( $\beta$-gal) plasmid (Promega Corporation) into $\mathrm{HaCaT}$ cells. Following $24 \mathrm{~h}$, the luciferase activity was measured using a dual-luciferase reporter assay system as recommended by the manufacturer (Promega Corporation).

miRNA microarray assay. Total RNA was purified from $\mathrm{HaCaT}$ cells that were UVB-irradiated with or without arctiin pretreatment using TRIzol reagent (Life Technologies) according to the manufacturer's instructions. The quality and concentration of the RNAs were analyzed using MaestroNano (Maestrogen, Las Vegas, NV, USA) and the integrity of the RNAs was determined using an Agilent 2100 Bioanalyzer (Agilent Technologies, Santa Clara, CA, USA). A microarray assay was performed with the SurePrint G3 Human V16 miRNA 8x60 K array (Agilent Technologies) as previously described (12) and the results were analyzed with GeneSpring GX software version 11.5 (Agilent Technologies). Differentially expressed miRNAs were selected using the criteria of a random variance t-test, $\mathrm{P}$-value $<0.05$ and an absolute fold change $>2$.

Bioinformatics analysis of miRNAs. Putative targets of each miRNA were identified using a DNA Intelligent Analysis (DIANA)-microT web-based bioinformatics program (http://diana.imis.athena-innovation.gr/DianaTools/index.php) (13). The involvement of putative target genes of each miRNA in the Kyoto Encyclopedia of Genes and Genomes (KEGG) pathways was determined using the web-based bioinformatics tool Database for Annotation, Visualization and Integrated Discovery (DAVID; http://david.abcc.ncifcrf.gov/home.jsp) (14).

Statistical analysis. All results were determined from three independent experiments and analyzed with the unpaired 

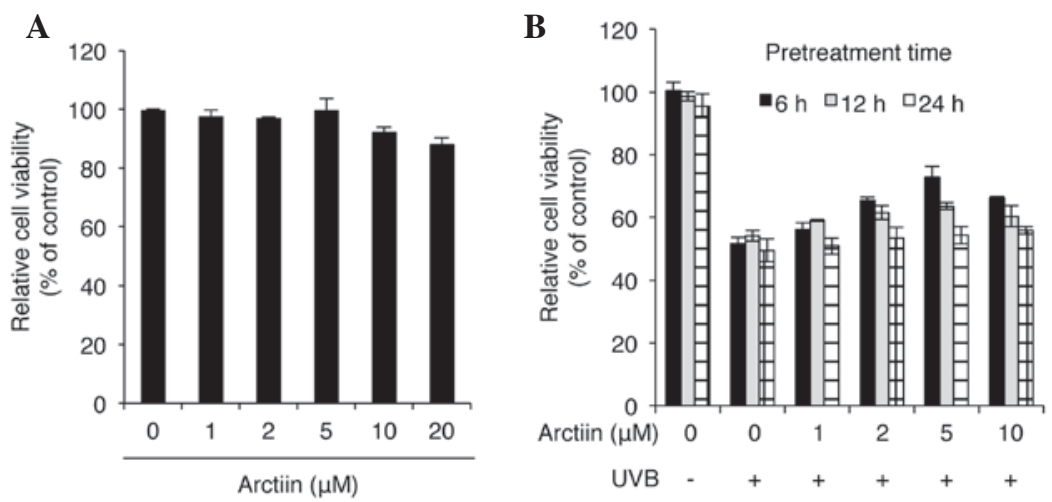

Figure 1. Arctiin inhibits UVB-mediated cytotoxicity in keratinocytes. (A) HaCaT keratinocytes were treated with different doses of arctiin for $24 \mathrm{~h}$ and cytotoxicity was analyzed by a WST-1 assay. (B) Cells were pretreated with various doses of arctiin for different durations prior to UVB irradiation. Cell viability was analyzed using the WST-1 assay. All data are expressed as the mean \pm standard deviation of three independent experiments. WST, water-soluble tetrazolium;_UVB, ultraviolet B; miRNA, microRNA.
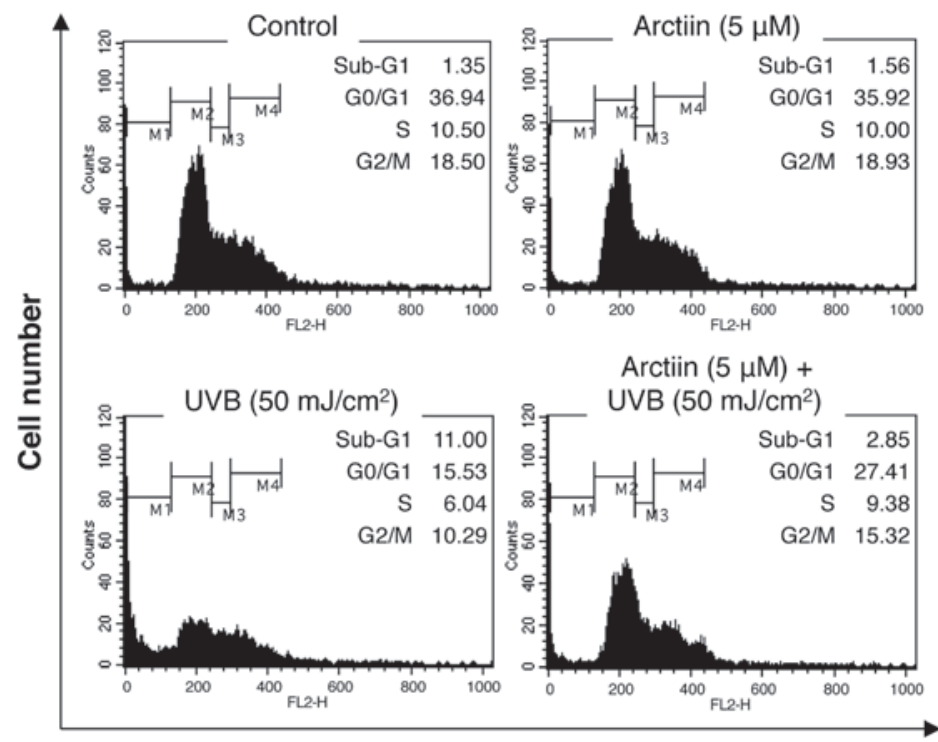

$\operatorname{Arctiin}(5 \mu \mathrm{M})+$ UVB $\left(50 \mathrm{~mJ} / \mathrm{cm}^{2}\right)$

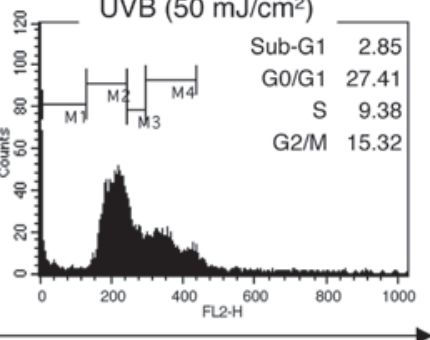

PI

Figure 2. Arctiin inhibits the induction of HaCaT cell death by UVB irradiation. HaCaT cells were pretreated with either dimethylsulfoxide or arctiin for $6 \mathrm{~h}$ prior to UVB irradiation and subjected to flow cytometric cell cycle analysis. The numbers represent the percentage of cells in sub-G1, G0/G1, S and G2/M phase. UVB, ultraviolet B; miRNA, microRNA; PI, propidium iodide.

Student's t-test. $\mathrm{P}<0.05$ was considered to indicate a statistically significant difference.

\section{Results}

Arctiin protects HaCaT cells against UVB damage. To determine the cytotoxicity of arctiin, a cell viability assay was performed using water-soluble tetrazolium salts (WSTs) following treatment of $\mathrm{HaCaT}$ cells with different doses of arctiin for $24 \mathrm{~h}$. Arctiin demonstrated no cytotoxicity at concentrations of $1-5 \mu \mathrm{M}$ and low cytotoxicity at 10 and $20 \mu \mathrm{M}$ (Fig. 1A). To investigate the UVB protective effect of arctiin, $\mathrm{HaCaT}$ cells were pretreated with arctiin at different doses and treatment durations prior to UVB $\left(50 \mathrm{~mJ} / \mathrm{cm}^{2}\right)$ irradiation and a cytotoxicity assay were performed. Significant UVB protection was observed in the cells pretreated with $5 \mu \mathrm{M}$ arctiin for $6 \mathrm{~h}$ (Fig. 1B). Although other pretreatment conditions also demonstrated UVB protective activities, cell viability was not significantly improved compared with UVB-irradiated control cells. These results identify arctiin as a novel UVB protective agent in keratinocytes.

Arctiin pretreatment antagonizes UVB-mediated HaCaT cell death. To determine the protective effect of arctiin on UVB-irradiated HaCaT cells, the cell cycle distribution was examined by PI staining and flow cytometry. UVB irradiation $\left(50 \mathrm{~mJ} / \mathrm{cm}^{2}\right)$ increased the cell population in the sub-G1 phase, indicating the induction of cell death (Fig. 2). Pretreatment with arctiin prior to UVB treatment markedly decreased the population of cells in sub-G1 phase, suggesting that arctiin affects the UVB-mediated cellular mechanisms that induce cell death (Fig. 2).

Arctiin pretreatment rescues UVB-induced deficiencies in wound healing and DNA repair. To examine whether arctiin pretreatment induced UVB resistance through the regulation 
A

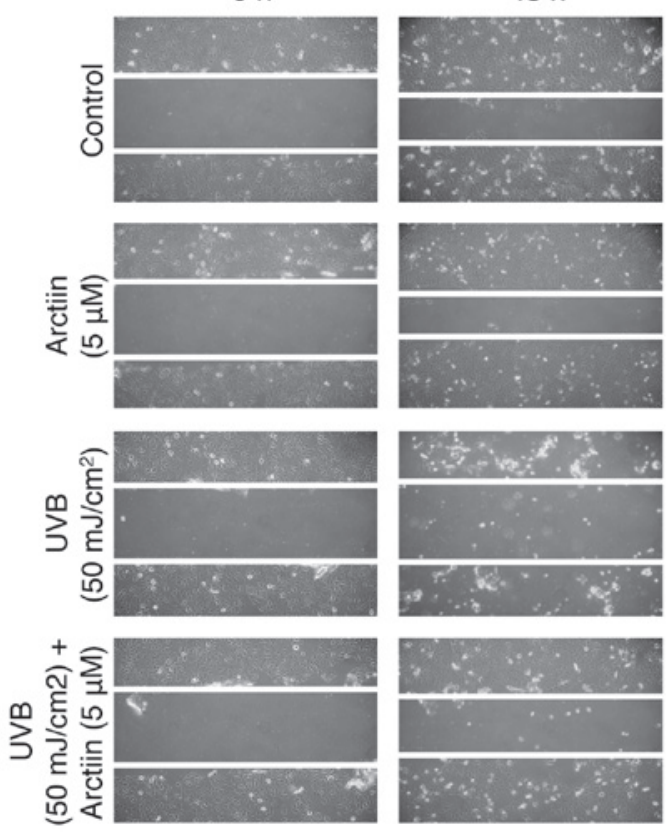

B

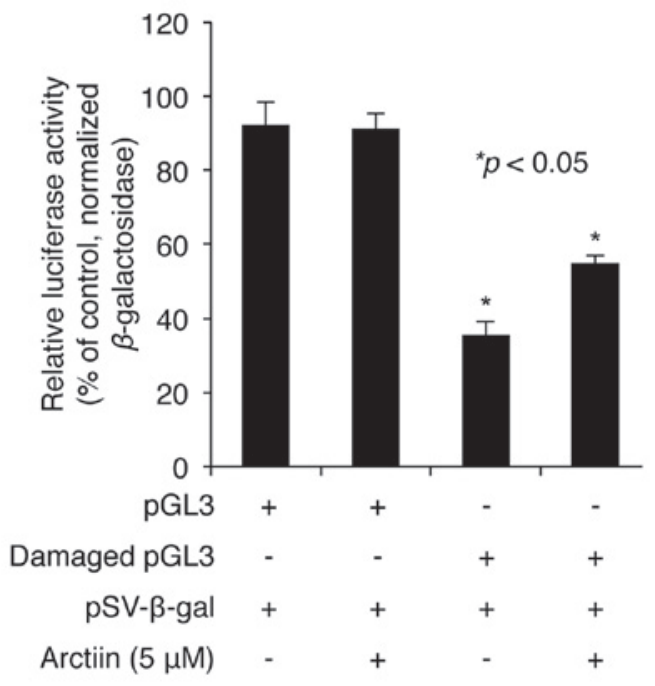

Figure 3. Effect of arctiin on scratch wound healing and DNA repair in UVB-irradiated HaCaT cells. (A) Arctiin rescues the UVB-induced wound-healing defect. Confluent cells were pretreated with arctiin and mechanically scratched with a pipette tip prior to UVB irradiation. Phase-contrast micrographs of scratched $\mathrm{HaCaT}$ cells were taken at 0 and $48 \mathrm{~h}$ following irradiation. (B) Arctiin enhances DNA repair. Cells were transfected with either untreated pGL3 or UV-irradiated pGL3 and then treated with arctiin for $24 \mathrm{~h}$. Following incubation, the luciferase activity in the cell lysate was measured and normalized to $\beta$-gal activity. Data are expressed as the mean \pm standard deviation of three independent experiments. ${ }^{*} \mathrm{P}<0.05$ vs. control cells (undamaged plasmid). UVB, ultraviolet B; miRNA, microRNA; gal, galactosidase.

of the cell migration associated with wound healing, a series of scratch assays were performed in the HaCaT cells. Firstly, marked closure of the scratch was demonstrated in the untreated control cells following $48 \mathrm{~h}$ incubation with DMSO vehicle (Fig. 3A, top panel). A similar extent of cell migration was observed in the cells treated with arctiin (5 $\mu \mathrm{M}$; Fig. 3A, second panel). However, UVB-irradiated cells demonstrated only a weak change in the extent of cell migration between 0 and $48 \mathrm{~h}$ (Fig. 3A, third panel). Of note, the UVB-mediated defect in cell migration was rescued by pretreatment with arctiin to a level of scratch closure similar to that in the control cells, suggesting that arctiin protects against the UVB-induced loss of migration in $\mathrm{HaCaT}$ cells (Fig. 3A, bottom panel).

To further examine the effect of arctiin on the repair of UV-induced DNA damage in HaCaT cells, a DNA repair assay was performed using the luciferase system and a damaged pGL3 luciferase plasmid that had been irradiated with UVC $\left(2000 \mathrm{~mJ} / \mathrm{cm}^{2}\right)$. The control or damaged pGL3 were co-transfected into $\mathrm{HaCaT}$ cells with the $\beta$-gal plasmid, followed by treatment with arctiin for $24 \mathrm{~h}$. In the absence of arctiin, the luciferase activity of damaged pGL3 was $\sim 40 \%$ of that measured for the undamaged control pGL3 (Fig. 3B). However, the decrease in luciferase activity for the damaged plasmid was significantly reduced in the cells treated with arctiin following transfection, implying that arctiin enhances DNA repair in $\mathrm{HaCaT}$ cells (Fig. 3B).

$U V B$ protective functions of arctiin are associated with changes in miRNA expression profiles in HaCaT cells. Next, the present study focused on exploring whether the UVB protective function of arctiin in $\mathrm{HaCaT}$ cells was associated with changes in miRNA expression profiles by miRNA microarray analysis. UVB radiation following arctiin pretreatment altered the expression levels of several miRNAs compared with UV treatment alone (Fig. 4A). Four miRNAs were significantly upregulated and 62 miRNAs were significantly downregulated $>2$-fold (Fig. 4B), implying that arctiin modulates the expression of several miRNAs to protect cells from UVB-induced $\mathrm{HaCaT}$ cell damage. The full list of dysregulated miRNAs is shown in Table I. miR-21-3p was the most highly upregulated (3.85-fold) and miR-513c-5p was the most downregulated (13.32-fold) miRNA during the arctiin-mediated UVB protective response in HaCaT cells.

Following this, the biological mechanisms by which altered miRNA expression mediates the UVB protection effect were investigated. miRNAs target mRNAs by directly interacting with complementary sequences, so in the present study, putative target genes of each miRNA were analyzed using the DIANA bioinformatics tool. The resultant target genes were then analyzed using the bioinformatics database DAVID. The results generated by the KEGG pathway-based enrichment analysis program in DAVID indicated that these miRNAs may be involved in the regulation of pathways in cancer, the cell cycle, Wnt and MAPK signaling pathways (Tables II and III). Targets of the majority of upregulated miRNAs had biological functions in pathways involved in cancer, the cell cycle and Wnt signaling as described in Table II, whereas the majority of the targets of downregulated miRNAs were involved in the regulation of the actin cytoskeleton, cancer pathways, and MAPK, Axon, ErbB and insulin signaling pathways (Table III). Previous studies 
Table I. Changes in miRNA expression in UVB-irratiated HaCaT cells pretreated with arctiin ${ }^{\mathrm{a}}$.

\begin{tabular}{|c|c|c|c|c|c|c|c|}
\hline Gene name & Fold change & Regulation & Chr & Gene name & Fold change & Regulation & Chr \\
\hline hsa-miR-125a-5p & 2.74 & Up & chr19 & hsa-miR-3656 & -4.21 & Down & $\operatorname{chr} 11$ \\
\hline hsa-miR-205-3p & 2.51 & Up & chr1 & hsa-miR-3663-3p & -3.31 & Down & $\operatorname{chr} 10$ \\
\hline hsa-miR-21-3p & 3.85 & Up & $\operatorname{chr} 17$ & hsa-miR-3665 & -3.01 & Down & $\operatorname{chr} 13$ \\
\hline hsa-miR-29b-1-5p & 2.81 & Up & $\operatorname{chr} 7$ & hsa-miR-3679-5p & -4.16 & Down & $\operatorname{chr} 2$ \\
\hline hsa-miR-1181 & -2.38 & Down & $\operatorname{chr} 19$ & hsa-miR-371a-5p & -2.26 & Down & $\operatorname{chr} 19$ \\
\hline hsa-miR-1202 & -3.40 & Down & chr6 & hsa-miR-3911 & -3.00 & Down & chr9 \\
\hline hsa-miR-1207-5p & -3.81 & Down & chr8 & hsa-miR-424-3p & -4.00 & Down & $\operatorname{chrX}$ \\
\hline hsa-miR-1224-5p & -2.70 & Down & $\operatorname{chr} 3$ & hsa-miR-4257 & -4.71 & Down & chr1 \\
\hline hsa-miR-1225-5p & -4.11 & Down & chr16 & hsa-miR-4270 & -4.41 & Down & chr3 \\
\hline hsa-miR-1234-3p & -2.90 & Down & chr8 & hsa-miR-4271 & -3.72 & Down & $\operatorname{chr} 3$ \\
\hline hsa-miR-1246 & -4.23 & Down & $\operatorname{chr} 2$ & hsa-miR-4281 & -3.08 & Down & $\operatorname{chr} 5$ \\
\hline hsa-miR-125a-3p & -2.86 & Down & chr19 & hsa-miR-4298 & -2.31 & Down & chr11 \\
\hline hsa-miR-1268a & -3.12 & Down & $\operatorname{chr} 15$ & hsa-miR-4299 & -3.21 & Down & chr11 \\
\hline hsa-miR-1275 & -3.94 & Down & chr6 & hsa-miR-4327 & -3.48 & Down & $\operatorname{chr} 21$ \\
\hline hsa-miR-1290 & -4.59 & Down & chr1 & hsa-miR-452-5p & -2.72 & Down & $\operatorname{chrX}$ \\
\hline hsa-miR-134 & -4.14 & Down & $\operatorname{chr} 14$ & hsa-miR-483-5p & -5.72 & Down & chr11 \\
\hline hsa-miR-135a-3p & -3.92 & Down & chr3 & hsa-miR-494 & -3.35 & Down & $\operatorname{chr} 14$ \\
\hline hsa-miR-1469 & -5.69 & Down & $\operatorname{chr} 15$ & hsa-miR-513a-5p & -5.23 & Down & $\operatorname{chrX}$ \\
\hline hsa-miR-150-3p & -3.28 & Down & chr19 & hsa-miR-513b & -4.68 & Down & $\operatorname{chrX}$ \\
\hline hsa-miR-188-5p & -4.11 & Down & $\operatorname{chrX}$ & hsa-miR-513c-5p & -13.32 & Down & $\operatorname{chrX}$ \\
\hline hsa-miR-1915-3p & -2.80 & Down & $\operatorname{chr} 10$ & hsa-miR-572 & -5.49 & Down & $\operatorname{chr} 4$ \\
\hline hsa-miR-1973 & -2.63 & Down & $\operatorname{chr} 4$ & hsa-miR-575 & -4.10 & Down & $\operatorname{chr} 4$ \\
\hline hsa-miR-28-5p & -2.20 & Down & chr3 & hsa-miR-629-3p & -2.33 & Down & $\operatorname{chr} 15$ \\
\hline hsa-miR-2861 & -4.08 & Down & $\operatorname{chr} 9$ & hsa-miR-630 & -4.37 & Down & $\operatorname{chr} 15$ \\
\hline hsa-miR-3127-5p & -2.00 & Down & $\operatorname{chr} 2$ & hsa-miR-638 & -4.40 & Down & $\operatorname{chr} 19$ \\
\hline hsa-miR-3141 & -2.33 & Down & chr5 & hsa-miR-642b-3p & -3.03 & Down & $\operatorname{chr} 19$ \\
\hline hsa-miR-3162-5p & -3.08 & Down & chr11 & hsa-miR-663a & -5.28 & Down & $\operatorname{chr} 20$ \\
\hline hsa-miR-3196 & -2.96 & Down & $\operatorname{chr} 20$ & hsa-miR-718 & -4.48 & Down & $\operatorname{chrX}$ \\
\hline hsa-miR-320c & -2.75 & Down & $\operatorname{chr} 18$ & hsa-miR-762 & -2.73 & Down & $\operatorname{chr} 16$ \\
\hline hsa-miR-345-5p & -10.76 & Down & chr14 & hsa-miR-765 & -6.70 & Down & chr1 \\
\hline hsa-miR-3646 & -2.75 & Down & $\operatorname{chr} 20$ & hsa-miR-874 & -3.09 & Down & $\operatorname{chr} 5$ \\
\hline hsa-miR-3648 & -4.46 & Down & $\operatorname{chr} 21$ & hsa-miR-939-5p & -2.01 & Down & chr8 \\
\hline hsa-miR-3652 & -8.13 & Down & $\operatorname{chr} 12$ & hsa-miR-940 & -2.39 & Down & $\operatorname{chr} 16$ \\
\hline
\end{tabular}

${ }^{\text {a }}$ selected miRNAs exhibiting a >2-fold expression change. Chr, chromosome; miR, microRNA; UVB, ultraviolet B; hsa, Homo sapiens.

have reported that these pathways are affected by UVB radiation $(2,15-17)$. In summary, these results identified the cellular processes involved in arctiin-mediated UVB protection.

\section{Discussion}

Keratinocytes are the predominant cell type in the outermost skin layer, the epidermis, and have a pivotal role in primary protection against environmental insults, including UV radiation. UV radiation is the major inducer of photoaging of keratinocytes and may result in cellular aging, senescence, apoptosis or cancer by inducing damage to intracellular molecules, in particular DNA. In the present study, biochemical and genetic analyses were utilized to identify the phytochemical arctiin as a novel photoprotective agent against UVB-mediated keratinocyte damage. The data revealed that arctiin itself had a low cytotoxicity in HaCaT cells. Notably, arctiin pretreatment reduced UVB-induced cytotoxicity and suppressed UVB-mediated cell death. It was also demonstrated that arctiin enhanced wound healing and DNA repair in UVB-exposed $\mathrm{HaCaT}$ keratinocytes.

Significant changes in miRNA expression profiles in cells that were pretreated with arctiin prior to UVB irradiation were observed, as compared with those in the cells that only received UVB irradiation, with 66 miRNAs exhibiting altered expression. Of note, almost all of the dysregulated miRNAs were downregulated (62/66) and only four miRNAs were upregulated. This expression pattern differs from the results of a previous study by our group in which the titrated extract of Centella asiatica (TECA) was demonstrated to protect keratinocytes against UVB-induced damage through upregulation of 46 miRNAs and downregulation of 36 miRNAs (12). In addition, Zhou et al (6) 
Table II. Functional annotation chart for miRNAs that were upregulated in arctiin-pretreated UVB-irradiated HaCaT keratinocytes.

\begin{tabular}{|c|c|c|c|c|c|}
\hline $\begin{array}{l}\text { miRNA } \\
\text { (Homo sapiens) }\end{array}$ & $\begin{array}{c}\text { Putative } \\
\text { target genes }\end{array}$ & KEGG pathway & $\begin{array}{l}\text { Genes involved } \\
\text { in the term }\end{array}$ & $\begin{array}{c}\% \text { of involved } \\
\text { genes/total genes }\end{array}$ & P-value \\
\hline \multirow[t]{2}{*}{ miR-125a-5p } & \multirow[t]{2}{*}{162} & Pathways in cancer & 8 & 4.9 & $3.60 \times 10^{-2}$ \\
\hline & & Cell cycle & 4 & 2.5 & $1.20 \times 10^{-1}$ \\
\hline \multirow[t]{3}{*}{ miR-205-3p } & \multirow[t]{3}{*}{944} & Pathways in cancer & 19 & 2 & $2.50 \times 10^{-1}$ \\
\hline & & MAPK signaling pathway & 17 & 1.8 & $1.70 \times 10^{-1}$ \\
\hline & & Wnt signaling pathway & 15 & 1.6 & $9.20 \times 10^{-3}$ \\
\hline \multirow[t]{4}{*}{$\operatorname{miR}-21-3 p$} & \multirow[t]{4}{*}{210} & CAMs & 7 & 3.3 & $4.70 \times 10^{-3}$ \\
\hline & & Ubiquitin mediated proteolysis & 6 & 2.9 & $2.30 \times 10^{-2}$ \\
\hline & & Long-term potentiation & 5 & 2.4 & $8.60 \times 10^{-3}$ \\
\hline & & Oocyte meiosis & 5 & 2.4 & $4.20 \times 10^{-2}$ \\
\hline \multirow[t]{4}{*}{$\operatorname{miR}-29 b-1-5 p$} & \multirow[t]{4}{*}{265} & Insulin signaling pathway & 5 & 1.9 & $8.50 \times 10^{-2}$ \\
\hline & & Cell cycle & 4 & 1.5 & $2.00 \times 10^{-1}$ \\
\hline & & Wnt signaling pathway & 4 & 1.5 & $2.90 \times 10^{-1}$ \\
\hline & & Jak-STAT signaling pathway & 4 & 1.5 & $3.00 \times 10^{-1}$ \\
\hline
\end{tabular}

CAMs, cell adhesion molecules; UVB, ultraviolet B; MAPK, mitogen-activated protein kinase; miR, microRNA; KEGG, Kyoto Encyclopedia of Genes and Genomes.

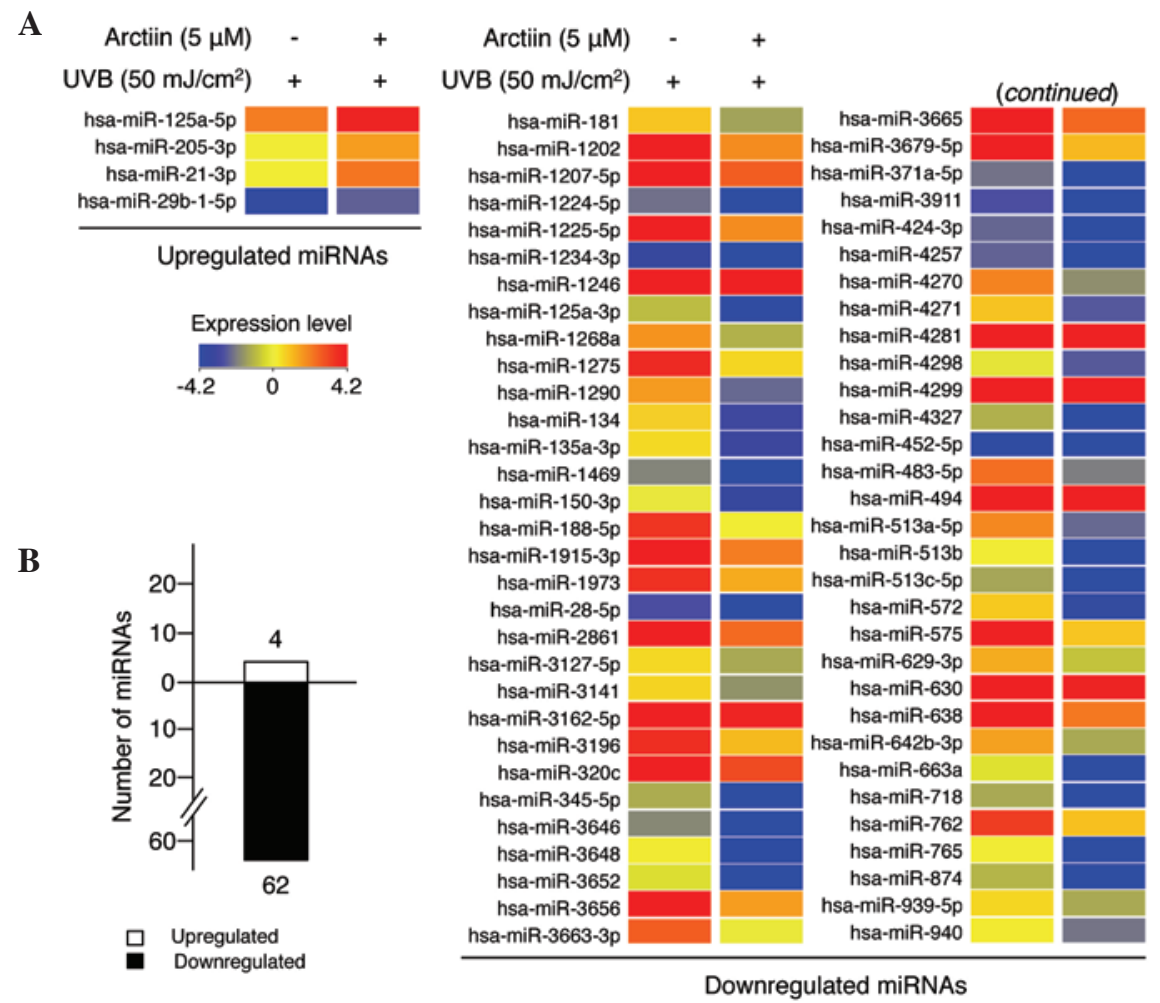

Figure 4. Comparison of miRNA expression in UVB-irradiated keratinocytes with or without arctiin pretreatment. (A) Results of miRNA microarray assays. $\mathrm{HaCaT}$ cells were treated with arctiin for $6 \mathrm{~h}$ prior to irradiation with UVB. Following $24 \mathrm{~h}$, total RNAs were isolated and subjected to microarray assay. Left panel, miRNAs that were upregulated >2-fold; right panel, miRNAs that were downregulated $>2$-fold. (B) Bar chart revealing the number of up- and downregulated miRNAs based on microarray data. UVB, ultraviolet B; miR/miRNA, microRNA; hsa, Homo sapiens.

recently characterized UVB-responsive miRNAs in human keratinocytes using microRNA microarray analysis and identified that the expression of 44 miRNAs was up- or downregulated by $>2$-fold compared with that in non-irradiated keratinocytes. There appears to be no correlation between the UVB-responsive
miRNAs and the arctiin-induced UVB protective miRNAs; however, certain miRNAs identified in the present study were also dysregulated in other studies. miR-3652 and miR-494, which were downregulated by 8.31- and 3.35-fold in the present study, were also downregulated in keratinocytes with TECA-induced 
Table III. Functional annotation chart for miRNAs that were downregulated in arctiin-pretreated UVB-irradiated HaCaT keratinocytes. ${ }^{a}$

\begin{tabular}{|c|c|c|c|c|c|}
\hline $\begin{array}{l}\text { miRNA } \\
\text { (Homo sapiens) }\end{array}$ & $\begin{array}{l}\text { Putative } \\
\text { target genes }\end{array}$ & KEGG pathway & $\begin{array}{l}\text { Genes involved } \\
\text { in the term }\end{array}$ & $\begin{array}{c}\% \text { of involved } \\
\text { genes/total genes }\end{array}$ & P-value \\
\hline \multirow[t]{3}{*}{$\operatorname{miR}-513 c-5 p$} & \multirow[t]{3}{*}{142} & Tight junction & 4 & 2.8 & $3.30 \times 10^{-2}$ \\
\hline & & Phosphatidylinositol signaling system & 3 & 2.1 & $5.80 \times 10^{-2}$ \\
\hline & & CAMs & 3 & 2.1 & $1.50 \times 10^{-1}$ \\
\hline \multirow[t]{6}{*}{ miR-345-5p } & \multirow[t]{6}{*}{94} & Axon guidance & 3 & 3.2 & $8.20 \times 10^{-2}$ \\
\hline & & Regulation of actin cytoskeleton & 3 & 3.2 & $1.90 \times 10^{-1}$ \\
\hline & & Type II diabetes mellitus & 2 & 2.1 & $1.60 \times 10^{-1}$ \\
\hline & & Arginine and proline metabolism & 2 & 2.1 & $1.80 \times 10^{-1}$ \\
\hline & & VEGF signaling pathway & 2 & 2.1 & $2.50 \times 10^{-1}$ \\
\hline & & TGF- $\beta$ signaling pathway & 2 & 2.1 & $2.80 \times 10^{-1}$ \\
\hline \multirow[t]{5}{*}{$\operatorname{miR}-3652$} & \multirow[t]{5}{*}{195} & Axon guidance & 5 & 2.6 & $3.30 \times 10^{-2}$ \\
\hline & & CAMs & 5 & 2.6 & $3.50 \times 10^{-2}$ \\
\hline & & Insulin signaling pathway & 5 & 2.6 & $3.80 \times 10^{-2}$ \\
\hline & & ErbB signaling pathway & 4 & 2.1 & $4.80 \times 10^{-2}$ \\
\hline & & MAPK signaling pathway & 4 & 2.1 & $4.70 \times 10^{-1}$ \\
\hline $\operatorname{miR}-765$ & 548 & Cytokine-cytokine receptor interaction & 11 & 2 & $2.00 \times 10^{-1}$ \\
\hline $\operatorname{miR}-483-5 p$ & 32 & Focal adhesion & 2 & 6.2 & $1.50 \times 10^{-1}$ \\
\hline miR-1469 & 2 & - & - & - & - \\
\hline $\operatorname{miR}-572$ & 6 & - & - & - & - \\
\hline miR-663a & 33 & - & - & - & - \\
\hline \multirow[t]{3}{*}{$\operatorname{miR}-513 a-5 p$} & \multirow[t]{3}{*}{980} & MAPK signaling pathway & 25 & 2.6 & $1.00 \times 10^{-2}$ \\
\hline & & Pathways in cancer & 24 & 2.4 & $1.30 \times 10^{-1}$ \\
\hline & & Regulation of actin cytoskeleton & 20 & 2 & $2.50 \times 10^{-2}$ \\
\hline \multirow[t]{6}{*}{ miR-1290 } & \multirow[t]{6}{*}{593} & Pathways in cancer & 17 & 2.9 & $4.00 \times 10^{-2}$ \\
\hline & & Focal adhesion & 14 & 2.4 & $7.90 \times 10^{-3}$ \\
\hline & & Insulin signaling pathway & 13 & 2.2 & $7.60 \times 10^{-4}$ \\
\hline & & Regulation of actin cytoskeleton & 12 & 2 & $6.30 \times 10^{-2}$ \\
\hline & & MAPK signaling pathway & 12 & 2 & $1.90 \times 10^{-1}$ \\
\hline & & ErbB signaling pathway & 11 & 1.9 & $2.80 \times 10^{-4}$ \\
\hline $\operatorname{miR}-718$ & 40 & - & - & - & - \\
\hline miR-3648 & 13 & - & - & - & - \\
\hline \multirow[t]{2}{*}{$\operatorname{miR}-4270$} & 423 & Axon guidance & 8 & 1.9 & $5.50 \times 10^{-3}$ \\
\hline & & Regulation of actin cytoskeleton & 8 & 1.9 & $6.70 \times 10^{-2}$ \\
\hline
\end{tabular}

${ }^{a}$ The list shows only the top 15 downregulated miRNAs displaying >2-fold expression changes. KEGG, Kyoto Encyclopedia of Genes and Genomes; CAMs, cell adhesion molecules; UVB, ultraviolet B; MAPK, mitogen-activated protein kinase; miR/miRNA, microRNA; VEGF, vascular endothelial growth factor; TGF, transforming growth factor; ErbB, epidermal growth factor receptor.

UVB protection (12). miR-125a-5p was previously reported to be expressed at significantly lower levels in UVB-irradiated mouse epidermis than in control epidermis (18), although the results of the present study demonstrated that the miRNA was significantly upregulated (2.74-fold). In addition, miR-1246 (4.23-fold downregulation in this study) was reported to be a target of the p53 transcription factor, which is significantly upregulated by UVB irradiation in keratinocytes (19), and miR-125a-3p (2.86-fold downregulation) has been demonstrated to reduce cell proliferation and migration by targeting Fyn kinase, which is activated by UVB irradiation in keratinocytes $(20,21)$. In summary, the microarray data of the present study suggested that although the dysregulated miRNAs were specifically induced by arctiin treatment, they may be involved in the regulation of general UVB-mediated cellular transduction. Although miR-513c-5p and miR-345-5p were the most highly dysregulated miRNAs in the present study (13.32- and 10.76-fold downregulated, respectively), their biological functions have not been reported. It is possible that these miRNAs also regulate UVB-mediated cytotoxicity in keratinocytes.

The biological significance of the dysregulated miRNAs identified in the present study was further examined. For this, 
the putative target genes of the miRNAs were predicted using DIANA, a web-based bioinformatics program, and the target genes involved in KEGG pathways were then analyzed using the DAVID database. The results indicated that the MAPK signaling as the pathway that was most affected by arctiin-induced UVB protection in keratinocytes. miR-205-3p, miR-3652, miR-513a-5p and miR-1290 were predicted to affect target genes involved in the MAPK pathway. Indeed, MAPK activation is one of the main signaling pathways activated by UVB exposure. In keratinocytes, UVB stimulates immediate activation of JNK, p38 MAPK and ERK1/2 (2). Activated JNK and p38 MAPK mediate both cell survival and death in UVB-irradiated keratinocytes (2). In addition, it was demonstrated that UVB-induced production of ROS (including $\mathrm{H}_{2} \mathrm{O}_{2}$ ) activates p38 MAPK and JNK signaling in keratinocytes, suggesting that UVB-induced ROS may affect signal transduction through MAPK pathways (2). Furthermore, it has been suggested that p38 MAPK and JNK have a pivotal role in the UVB-induced inflammatory response by regulating cyclooxygenase-2 (COX-2) activity in keratinocytes (2). Of note, $\mathrm{UV}$ radiation does not regulate the mRNA levels of $J N K, p 38$ $M A P K$ and $E R K 1 / 2$, but rather the levels of post-translational modification by protein phosphorylation. This indicates that the activation of MAPK proteins by UVB radiation is not transcriptionally induced (2). However, recent studies have demonstrated that post-transcriptional regulators, including miRNAs, may regulate MAPK activity. Although the miRNAs do not directly regulate mRNA levels of MAPKs, upstream or downstream members of MAPK signaling pathways are post-transcriptionally regulated by miRNAs. Zhang et al (22) demonstrated that miR-451 regulates p38 MAPK signaling by targeting of Ywhaz, and Antoon et al (23) demonstrated that increased p38 MAPK activity results in part from differential miRNA expression. Therefore, arctiin-induced UVB protective miRNAs may regulate members of MAPK signaling pathways, rather than the mRNAs of MAPKs themselves.

In conclusion, the present study suggested that arctiin may be a novel photoprotective agent that confers resistance to UVB-mediated keratinocyte damage by altering miRNA expression profiles. In particular, the MAPK signaling pathway appears to be a major target of the miRNAs that are affected by arctiin. Further studies are required to determine the exact mechanisms by which arctiin induces a UVB protective effect in keratinocytes.

\section{Acknowledgements}

The authors are grateful to all other members of Coreana Cosmetics Co., Ltd. for their support. This paper was supported by the KU Research Professor Program of Konkuk University and grant from the Ministry of Science, ICT and Future Planning (no. 20110028646) of the Republic of Korea.

\section{References}

1. Wondrak GT, Jacobson MK and Jacobson EL: Endogenous UVA-photosensitizers: mediators of skin photodamage and novel targets for skin photoprotection. Photochem Photobiol Sci 5: 215-237, 2006.
2. Muthusamy V and Piva TJ: The UV response of the skin: a review of the MAPK, NFkappaB and TNFalpha signal transduction pathways. Arch Dermatol Res 302: 5-17, 2010.

3. Rivetti di Val Cervo P, Lena AM, Nicoloso M, et al: p63-microRNA feedback in keratinocyte senescence. Proc Natl Acad Sci USA 109: 1133-1138, 2012.

4. Hildebrand J, Rütze M, Walz N, et al: A comprehensive analysis of microRNA expression during human keratinocyte differentiation in vitro and in vivo. J Invest Dermatol 131: 20-29, 2011.

5. Yang X, Wang J, Guo SL, et al: miR-21 promotes keratinocyte migration and re-epithelialization during wound healing. Int J Biol Sci 7: 685-690, 2011.

6. Zhou BR, Xu Y, Permatasari F, et al: Characterization of the miRNA profile in UVB-irradiated normal human keratinocytes. Exp Dermatol 21: 317-319, 2012.

7. Guo Z, Zhou B, Liu W, et al: miR-23a regulates DNA damage repair and apoptosis in UVB-irradiated $\mathrm{HaCaT}$ cells. J Dermatol Sci 69: 68-76, 2013.

8. Lee S, Shin S, Kim H, et al: Anti-inflammatory function of arctiin by inhibiting COX-2 expression via NF-kB pathways. J Inflamm (Lond) 8: 16, 2011.

9. Hirose M, Yamaguchi T, Lin C, et al: Effects of arctiin on PhIP-induced mammary, colon and pancreatic carcinogenesis in female Sprague-Dawley rats and MeIQx-induced hepatocarcinogenesis in male F344 rats. Cancer Lett 155: 79-88, 2000.

10. Hayashi K, Narutaki K, Nagaoka Y, Hayashi T and Uesato S: Therapeutic effect of arctiin and arctigenin in immunocompetent and immunocompromised mice infected with influenza $A$ virus. Biol Pharm Bull 33: 1199-1205, 2010.

11. Matsuzaki Y, Koyama M, Hitomi T, et al: Arctiin induces cell growth inhibition through the down-regulation of cyclin D1 expression. Oncol Rep 19: 721-727, 2008.

12. An IS, An S, Choe TB, et al: Centella asiatica protects against UVB-induced HaCaT keratinocyte damage through microRNA expression changes. Int J Mol Med 30: 1349-1356, 2012.

13. Maragkakis M, Reczko M, Simossis VA, et al: DIANA-microT web server: elucidating microRNA functions through target prediction. Nucleic Acids Res 37: W273-W276, 2009.

14. Huang da W, Sherman BT and Lempicki RA: Systematic and integrative analysis of large gene lists using DAVID bioinformatics resources. Nat Protoc 4: 44-57, 2009.

15. Misovic M, Milenkovic D, Martinovic T, Ciric D, Bumbasirevic V and Kravic-Stevovic T: Short-term exposure to UV-A, UV-B, and UV-C irradiation induces alteration in cytoskeleton and autophagy in human keratinocytes. Ultrastruct Pathol 37: 241-248, 2013

16. Madson JG and Hansen LA: Multiple mechanisms of Erbb2 action after ultraviolet irradiation of the skin. Mol Carcinog 46: 624-628, 2007.

17. Yamada T, Hasegawa S, Inoue $\mathrm{Y}$, et al: $\mathrm{Wnt} / \beta$-catenin and kit signaling sequentially regulate melanocyte stem cell differentiation in UVB-induced epidermal pigmentation. J Invest Dermatol 133: 2753-2762, 2013.

18. Zhou BR, Xu Y and Luo D: Effect of UVB irradiation on microRNA expression in mouse epidermis. Oncol Lett 3: 560-564, 2012

19. Henseleit U, Zhang J, Wanner R, Haase I, Kolde G and Rosenbach T: Role of p53 in UVB-induced apoptosis in human HaCaT keratinocytes. J Invest Dermatol 109: 722-727, 1997.

20. Ninio-Many L, Grossman H, Shomron N, Chuderland D and Shalgi R: microRNA-125a-3p reduces cell proliferation and migration by targeting Fyn. J Cell Sci 126: 2867-2876, 2013.

21. He Z, Cho YY, Ma WY, Choi HS, Bode AM and Dong Z: Regulation of ultraviolet B-induced phosphorylation of histone $\mathrm{H} 3$ at serine 10 by Fyn kinase. J Biol Chem 280: 2446-2454, 2005.

22. Zhang Z, Luo X, Ding S, et al: microRNA-451 regulates $\mathrm{p} 38$ MAPK signaling by targeting of Ywhaz and suppresses the mesangial hypertrophy in early diabetic nephropathy. FEBS Lett 586: 20-26, 2012.

23. Antoon JW, Nitzchke AM, Martin EC, et al: Inhibition of p38 mitogen-activated protein kinase alters microRNA expression and reverses epithelial-to-mesenchymal transition. Int J Oncol 42: 1139-1150, 2013. 